\title{
Complicações arteriais da síndrome do desfiladeiro torácico
}

\author{
Arterial complications of thoracic outlet syndrome
}

\section{Fernando Thomazinho, Wander Eduardo Sardinha, Jose Manoel da Silva Silvestre, Domingos de Morais Filho, Fernando Motta*}

\section{Resumo}

As manifestações clínicas da síndrome do desfiladeiro torácico são predominantemente neurológicas, sendo as complicações arteriais raras, mas potencialmente graves. Entre elas, devemos citar os aneurismas com complicações embólicas e a trombose. Os autores relatam o caso de uma mulher de 37 anos com costela cervical bilateral que apresentou embolia no membro superior direito originada de um aneurisma pós-estenótico da artéria subclávia direita, além de apresentar ectasia da subclávia esquerda também por compressão.

Palavras-chave: Síndrome do desfiladeiro torácico, costela cervical, complicações arteriais.

\section{Introdução}

A expressão "síndrome do desfiladeiro torácico" (thoracic outlet syndrome - SDT) foi utilizada pela primeira vez por Peet ${ }^{1}$ para descrever os pacientes com sintomas neurovasculares relativos aos possíveis locais de compressão desse feixe, que basicamente são três: triângulo intercostoescalênico, espaço costoclavicular e espaço retrocoracopeitoral.

Acredita-se que, para ocorrer a síndrome, é necessária a combinação de dois fatores: 1) estreitamento anatômico; 2) algum tipo de traumatismo que desencadeie os sintomas ${ }^{2}$.

A compressão no triângulo intercostoescalênico, conhecida como síndrome dos escalenos, apresenta sintomas neurológicos e arteriais, não havendo sintomas venosos, pois a veia subclávia não está contida nesse triângulo. A síndrome de compressão costoclavicular pode comprimir qualquer estrutura do feixe. A compressão

\section{Abstract}

The clinical manifestations of thoracic outlet syndrome are mainly neurological. Although arterial complications are rare, they are potentially severe. Among these are aneurysms associated with embolism and thrombosis. The authors report a case of a 37 year-old woman with bilateral cervical rib that developed embolism in the right upper limb from a poststenotic right subclavian artery aneurysm and dilatation of the left subclavian artery, both due to compression.

Keywords: Thoracic outlet syndrome, cervical rib, arterial complications.

no espaço retrocoracopeitoral, ou síndrome do peitoral menor, é rara, e o tratamento é basicamente clínico.

Há outros locais possíveis de compressão ${ }^{3}$, com manifestações semelhantes. Entre eles, podemos citar a síndrome do mediano, onde ocorre compressão da artéria axilar entre os feixes do plexo braquial, e a síndrome da arcada de Langer, onde ocorre compressão do feixe vasculonervoso por músculo anômalo, o músculo áxilo-peitoral.

Os sintomas principais são dores e parestesias, que ocorrem espontaneamente ou são provocadas ou exacerbadas por movimentos que diminuem as dimensões dos potenciais espaços de compressão. Sua distribuição é mais freqüente na face volar do antebraço e dos últimos dois dedos.

As publicações históricas iniciais descreviam quase que exclusivamente manifestações vasculares ${ }^{4}$. Com o tempo, houve maior conhecimento dessa síndrome;

* Departamento de Clínica Cirúrgica, Serviço de Cirurgia Vascular, Hospital Universitário Estadual do Norte do Paraná (HURNP), Universidade Estadual de Londrina, Londrina, PR.

Não foram declarados conflitos de interesse associados à publicação deste relato de caso.

Artigo submetido em 27.08.07, aceito em 05.03.08. 


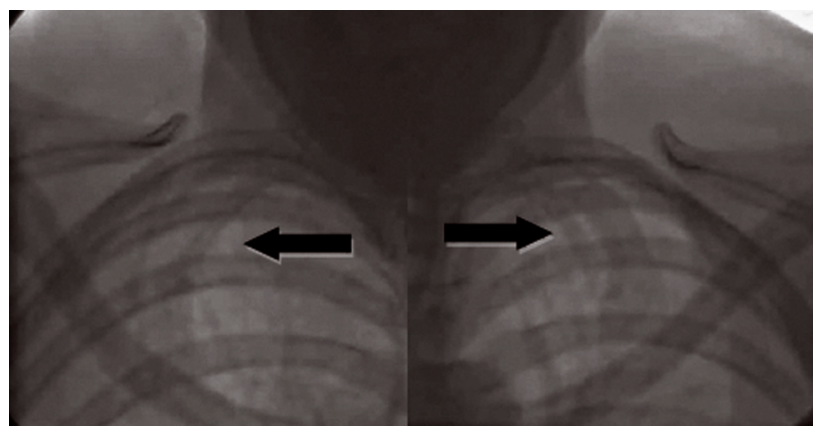

Figura 1 - Radiografia de tórax na incidência apicolordótica (setas indicam costela cervical completa bilateral)

notou-se que os sintomas neurológicos eram muito mais freqüentes e, atualmente, sabe-se que constituem a maioria dos casos, correspondendo a aproximadamente $95 \%$ das queixas clínicas. A presença de alterações na pesquisa das pulsações arteriais, com manobras que simulam compressão, não indicam necessariamente que exista lesão arterial estabelecida.

As complicações arteriais são raras, porém potencialmente mais graves que as manifestações neurológicas, podendo levar a seqüelas significativas.

Os autores relatam o caso de uma paciente com oclusão arterial aguda no membro superior direito por embolia originada de um aneurisma pós-estenótico como complicação da SDT.

\section{Relato de caso}

Paciente do sexo feminino, 36 anos de idade, com queixa de cianose, palidez e parestesias no membro superior direito há 3 dias. Negava queixa de parestesia nos membros superiores previamente. Não apresentava nenhuma comorbidade, não era tabagista e não fazia uso de anticoncepcional oral.

No exame físico inicial, a paciente apresentava palidez de dedos e mão direita com hiperemia reativa. Não apresentava pulsos radial e ulnar no membro superior direito, pulsos normais no membro contralateral e nos membros inferiores. Manobras de Adson realizadas bilateralmente resultaram positivas.

$\mathrm{Na}$ radiografia toracocervical na incidência apicolordótica, evidenciou-se costela cervical bilateral completa e articulada na primeira costela, bilateralmente (Figura 1).

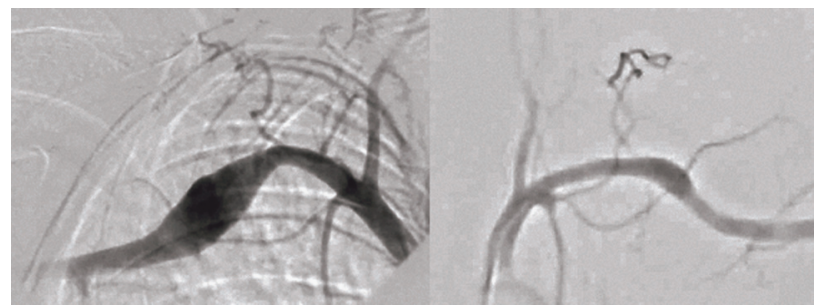

Figura 2 - Arteriografia de troncos supra-aórticos revelando aneurisma da artéria subclávia direita e ectasia da artéria subclávia esquerda

Foi realizado eco-Doppler dos membros superiores, onde foi evidenciada oclusão das artérias radial e ulnar no terço proximal do antebraço direito. Também mostrou positividade para testes de compressão arterial através da manobra dos escalenos, com diminuição expressiva da velocidade sistólica de pico na artéria axilar.

$\mathrm{Na}$ angiografia digital por punção femoral, foi evidenciado aneurisma de subclávia direita, com oclusão embólica das artérias do antebraço, e observada ectasia da artéria subclávia esquerda (Figura 2).

A paciente foi submetida à cirurgia com via de acesso por cervicotomia anterior direita em colar em um primeiro tempo. Realizou-se ressecção de costela cervical direita, com escalenectomia anterior e exérese do aneurisma da artéria subclávia direita (Figura 3) com enxerto interposto de veia safena magna, apresentando evolução satisfatória e sem complicações no pós-operatório.

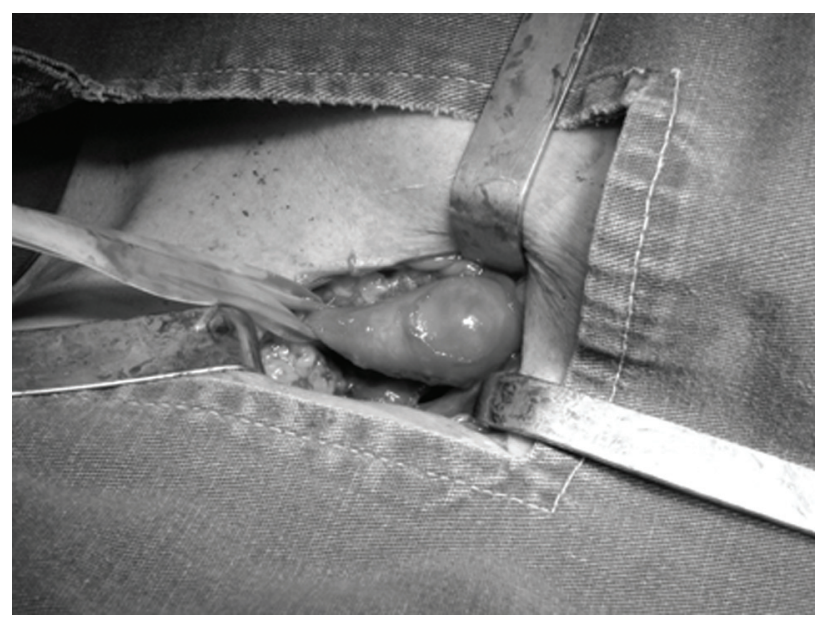

Figura 3 - Imagem intra-operatória do aneurisma da artéria subclávia direita 
Em um segundo tempo, após 30 dias, foi submetida à ressecção de costela cervical esquerda com escalenectomia anterior. Apresentou boa evolução e nenhuma complicação.

\section{Discussão}

As costelas cervicais e a primeira costela anômala são condições raras, presentes em aproximadamente 1\% da população e em $4,5 \%$ dos pacientes com $\mathrm{SDT}^{5}$. Geralmente, são achados de exames de imagem, como radiografias de tórax. A maioria é completamente assintomática, poucas vezes necessitando de tratamento. As costelas cervicais e primeira costela anômala podem apresentar complicações que constituem ameaças potenciais ao membro superior, necessitando de acompanhamento regular.

Segundo Sanders et al. ${ }^{5}$, o início dos sintomas é precedido por trauma cervical em $80 \%$ dos casos, e $20 \%$ dos casos ocorrem espontaneamente. Entretanto, num subgrupo de 12 pacientes que possuíam costela cervical completa, a incidência de sintomas espontâneos chegou a $50 \%$. De um total de 40 pacientes com costela cervical, em 11 casos ela media de 1 a $2 \mathrm{~cm}$; de 2 a $5 \mathrm{~cm}$ em 17 casos; e eram completas nos 12 pacientes restantes. Nos casos de costela completa, três eram articuladas diretamente com a primeira costela, e o restante se inseria na última por meio de um ligamento fibroso.

O tipo de costela cervical é de grande significado nas complicações arteriais. Ficou bem estabelecido, depois do estudo de Gruber $^{6}$, que as costelas curtas (tipo I) e as incompletas (tipo II) produzem preferencialmente complicações neurológicas, enquanto que as longas ou completas (tipo III) apresentam complicações arteriais ${ }^{6}$. Sanders et al. ${ }^{7}$ confirmaram os mesmos achados. Short et al. ${ }^{4}$ mostraram que $75 \%$ dos pacientes com costela cervical incompleta tiveram o início dos sintomas associados com trauma cervical, enquanto que nos pacientes com costela cervical completa apenas $50 \%$ dos casos tiveram essa associação.

A paciente em questão possuía costela cervical bilateral completa (tipo III), com complicações arteriais, aneurisma da artéria subclávia direita e ectasia da artéria subclávia esquerda. Possivelmente, a dilatação mais severa à direita deve-se ao fato de a paciente em questão ser destra, pois a maioria dos trabalhos tenta correlacionar a SDT com trauma do membro superior ${ }^{8}$.

As manifestações clínicas compreendem uma ampla gama de sintomas, sendo que nem todos estão necessariamente presentes no quadro clínico de cada paciente. A maioria dos sintomas é de compressão neurológica, e estes se manifestam como dor na região cervical, ombro, tórax anterior, cotovelo, braço, antebraço e cefaléia occipital. É comum referirem parestesias em dedos, mais freqüentemente no quarto e no quinto, devido a compressão das raízes mais inferiores do nervo ulnar, diminuição de força em mão e braço; geralmente, ocorre agravamento dos sintomas com a elevação do membro. Podem ser encontrados ainda: redução de temperatura, fenômeno de Raynaud e sudorese aumentada em região de mão. A incidência de cada sintoma é similar à dos pacientes que não possuem costela cervical.

As lesões arteriais na SDT são geralmente devidas a anormalidades ósseas. Achados na literatura correlacionam à presença da costela cervical em 70 a 100\% dos casos. Nesses pacientes, a artéria subclávia passa sobre a costela cervical (que a comprime no seu aspecto inferior) e produz uma lesão intimal com ou sem dilatação pós-estenótica ${ }^{9}$. Não raramente, esses pacientes apresentam complicações embólicas em sua evolução, que são o aspecto mais debilitante da patologia e de difícil tratamento. Complicações arteriais tendem a ser tratadas tardiamente em relação aos seus equivalentes neurológicos. De fato, na maior parte das vezes, o problema de base não é reconhecido até que o tromboembolismo tenha ocorrido, seja por uma lesão intimal do segmento subclavicular ou por dilatação pós-estenótica ou aneurisma ${ }^{10}$.

A presença de primeira costela anômala e de alterações musculares aumenta a probabilidade de lesão arterial e fenômenos embólicos. A primeira costela anômala leva a alterações semelhantes às da costela cervical, porém afeta a artéria no seu aspecto superior. A hipertrofia do músculo escaleno anterior em atletas está associada com comprometimento da artéria subclávia, e sua oclusão, com embolização dista $1^{10}$.

A necessidade de se detectar precocemente as lesões causadas por êmbolos num estágio precoce é de suma importância, e todos os pacientes com anormalidades 
de primeira costela ou com costela cervical devem ser acompanhados regularmente com ultra-sonografia Doppler do segmento subclávio-axilar ${ }^{10}$. Todo segmento estenótico ou presença de aneurisma deve ser avaliado posteriormente com uma angiografia para programação de uma intervenção cirúrgica.

Vários pacientes que procuram assistência médica com isquemia severa apresentam história prévia de episódios de alerta. Em um paciente com história de fenômeno de Raynaud unilateral (especialmente no território radial $)^{11}$ e em qualquer paciente que experimenta uma dor súbita em polpa digital com alterações vasomotoras (palidez ou cianose), deve ser suspeitado um evento embólico e deve ser iniciada investigação com ecoDoppler e angiografia do desfiladeiro torácico ${ }^{12}$.

As radiografias de tórax e coluna cervical são de extrema valia para revelar alterações ósseas. A tomografia computadorizada pode ser útil no diagnóstico de SDT, particularmente naqueles pacientes em que as radiografias são normais ${ }^{13}$. A ressonância magnética tem se mostrado eficaz em demonstrar bandas fibróticas e desvio do plexo braquial em pacientes sem costela cervical $^{14}$. A avaliação eletrodiagnóstica é útil para diagnóstico na compressão do carpo e no canal de Guyon, mas no diagnóstico da SDT sofre alterações devido à posição em que é realizado o teste ${ }^{15,16}$. Komanetsky et al., estudando 21 doentes com SDT e 23 controles, em posição relaxada e forçada, não consideraram esse teste útil para o diagnóstico ${ }^{17}$.

O tratamento dessa enfermidade pode ser conservado com fisioterapia, repouso da atividade que originou os sintomas e analgesia quando a síndrome for principalmente de comprometimento neurológica ${ }^{1}$. Em casos de falha no tratamento clínico (após 6 meses sem melhora ou reaparecimento dos sintomas $)^{1}$, nãocontrole da dor e da parestesia, além de acometimento vascular (palidez, pulso diminuído, cianose, fenômeno de Raynaud ${ }^{11}$, claudicação do membro ou dor em repouso ou lesões tróficas) ${ }^{1}$, deve ser indicado o tratamento cirúrgico.

Existem duas técnicas cirúrgicas para correção: via supraclavicular e via transaxilar ${ }^{5,7,18}$. A primeira consiste em um acesso que pode ser infra ou supraclavicular com incisão do platisma, escalenectomia anterior e média, além da ressecção da primeira costela com exposição do plexo braquial e da artéria e veia subclávias $^{5,7,18,19}$. A segunda usa via de acesso através da axila ipsilateral, com ressecção da primeira costela, porém preservando o músculo escaleno ${ }^{19,20}$.

Estudos têm demonstrado que as taxas de sucesso têm sido similares entre as técnicas ${ }^{5,7}$, muito embora a via de acesso supraclavicular permita uma melhor visualização do músculo escaleno. Além disso, quando o músculo escaleno apresenta alguma anormalidade que possa ser responsável pela síndrome, é possível incisá-lo neste momento; por outro lado, utilizando-se a via transaxilar, tal procedimento seria feito em outro tempo cirúrgico.

Uma das principais complicações da cirurgia tem sido a lesão do nervo frênico ${ }^{5}$ e que normalmente regride em 3 meses na maioria dos casos. Outras complicações que têm sido relatadas são as lesões da veia e artéria subclávias. A primeira é muito mais freqüente que a segunda ${ }^{5,7,19,20}$. O pneumotórax também tem sido relatado, sendo mais freqüente quando se utiliza a via transaxilar $^{7,19,21}$, porém raras vezes foi necessária toracocentese.

No caso da paciente em questão, por se tratar de uma SDT vascular (comprometimento arterial puro) por compressão de uma costela cervical verdadeira, foi realizada uma via de acesso supraclavicular com ressecção da costela cervical, associada à escalenectomia à direita. Além disso, foi realizada ressecção do aneurisma com enxerto interposto de veia safena. Essa via foi optada pela exposição irrestrita do triângulo interescalênico e acesso bom para ressecção da costela cervical ${ }^{7,12}$. A mesma técnica foi utilizada para a ressecção da costela cervical no lado esquerdo, sem a necessidade de resseção arterial, pois a artéria subclávia desse lado encontrava-se apenas ectasiada.

A SDT é uma doença incomum que possui uma anatomia complexa e uma grande variação anatômica das estruturas, que deve ser manejada por equipe especializada e cirurgião vascular treinado nesse tipo de cirurgia.

\section{Referências}

1. Peet RM, Hendriksen JD, Anderson TP, Martin GM. Thoracic-outlet syndrome: evaluation of therapeutic exercise program. Proc Staff Meet Mayo Clin. 1956;31:281-7. 
2. Sanders RJ, Roos DB. The surgical anatomic of the scalene triangle. Contemp Surg. 1989;35:11-6.

3. Baptista-Silva JCC, Prates JC, Francisco Jr. J, Miranda Jr. F, Burihan E. Ligamentos do aparelho suspensor da pleura: morfologia e relação com o plexo braquial e os vasos subclávios. Rev Col Bras Cir. 1992;19:51-7.

4. Short DW. The subclavian artery in 16 patients with complete cervical ribs. J Cardiovasc Surg (Torino). 1975;16:13541.

5. Sanders RJ, Hammond SL. Management of cervical ribs and anomalous first ribs causing neurogenic thoracic outlet syndrome. J Vasc Surg. 2002;36:51-6.

6. Gruber W. Ueber die Halsrippen des Menshen mit verglerchendanatomischen Bermerkungen. St Petersburg: Memoires de l'academie Imperial Scientia; 1869.v.2,p.7-27.

7. Sanders RJ, Pearce WH. The treatment of thoracic outlet syndrome: a comparison of different operations. J Vasc Surg. 1989;10:626-34.

8. Nijhuis HH, Müller-Wiefel H. Occlusion of the brachial artery by thrombus dislodged from a traumatic aneurysm of the anterior humeral circumflex artery. J Vasc Surgery. 1991;13:408-11.

9. Durham JR, Yao JS, Pearce WH, Nuber GM, McCarthy WJ 3rd. Arterial injuries in the thoracic outlet syndrome. J Vasc Surg. 1995;21:57-69; discussion 70.

10. Cormier JM, Amrane M, Ward A, Laurian C, Gigou F. Arterial complications of the thoracic outlet syndrome: fifty-five operative cases. J Vasc Surg. 1989;9:778-87.

11. Bouhoutsos J, Morris T, Martin P. Unilateral Raynaud's phenomenon in the hand and its significance. Surgery. 1977;82:547-51.

12. Cruz M, Matos AA, Saldanha T, Branco JC. Angiografia como método diagnóstico da síndrome do desfiladeiro torácico neurovascular: a propósito de um caso. Rev Bras Reumatol. 2003;4:267-71.

13. Mackinnon SE, Novak CB. Evaluation of the patient with thoracic outlet syndrome. Semin Thorac Cardiovasc Surg. 1996;8:190-200.
14. Urschel JD, Hameed SM, Grewal RP. Neurogenic thoracic outlet syndromes. Postgrad Med J. 1994;70:785-9.

15. Machleder HI, Moll F, Nuwer M, Jordan S. Somatosensory evoked potentials in the assessment of thoracic outlet syndrome. J Vasc Surg. 1987;6:177-84.

16. Borg K, Persson HE, Lindblom U. Thoracic outlet syndome: diagnostic value of sensibility testing, vibratory thresholds and somatosensory evoked potentials at rest and during perturbation with abduction and external rotation of the arm. Proceedings from the Fifth World Congress on Pain, Amsterdam, Elsevier 1988. p. 144-50.

17. Komanetsky RM, Novak CB, Mackinnon SE, Russo MH, Padberg AM, Louis S. Somatosensory evoked potentials fail to diagnose thoracic outlet syndrome. J Hand Surg [Am]. 1996;21:662-6.

18. Reilly LM, Stoney RJ. Supraclavicular approach for thoracic outlet decompression. J Vasc Surg. 1988;8:329-34.

19. Sanders RJ, Cooper MA, Hammond SL, Weinstein ES. Neurogenic thoracic outlet syndrome. In: Rutherford R, editor. Vascular surgery. 5th ed. Philadelphia: WB Saunders; 1999. p. 1184-200.

20. Roos DB. Transaxillary first rib resection for thoracic outlet syndrome. In: Yao JST, Pearce WH, editores. Techniques in vascular and endovascular surgery. Stanford: Appleton e Lange; 1997. p. 531-8.

21. Makhoul RG, Machleder HI. Developmental anomalies at the thoracic outlet: An analysis of 200 consecutive cases. J Vasc Surg. 1992;16:534-42; discussion 542-5.

\section{Correspondência:}

Wander Eduardo Sardinha

Rua Borba Gato, 1079, Jardim Ipiranga

CEP 86010-630 - Londrina, PR

Tel.: (43) 3322.1411, (43) 9911.3015

E-mail: wandersardinha@uol.com.br

\section{O conteúdo do J Vasc Bras está disponivel em português e em inglês no site do Jornal Vascular Brasileiro em www.jvascbr.com.br}

